\title{
MULTIPLIERS ON SUBSEMIGROUPS OF THE REAL LINE
}

\author{
HUNG T. DINH
}

(Communicated by Palle E. T. Jorgensen)

\begin{abstract}
We show that multipliers on subsemigroups of the real line can be extended to multipliers on groups.
\end{abstract}

Multipliers on subsemigroups of the real line arise from the index theory of semigroups of endomorphisms of type I factors [1, 2, 4, 5]. Although multipliers on groups have been extensively studied [7], little is known about multipliers on semigroups.

Let $G$ be a subgroup of $\mathbf{R}$ and $G^{+}$be the semigroup of nonnegative elements of $G$. A multiplier of $G^{+}$(resp. $G$ ) is a Borel-measurable function $f: G^{+} \times$ $G^{+} \rightarrow \mathbf{T}$ (resp. $f: G \times G \rightarrow \mathbf{T}$ ) satisfying

$$
\begin{aligned}
f(r, s) f(r+s, t) & =f(s, t) f(r, s+t), \\
f(t, 0) & =f(0, t)=1
\end{aligned}
$$

for every $r, s, t \in G^{+}$(resp. $G$ ).

We say that two multipliers $f_{1}$ and $f_{2}$ on $G^{+}$are similar if there is a measurable function $a: G^{+} \rightarrow \mathrm{T}$ satisfying

$$
f_{2}(s, t)=\frac{a(s+t)}{a(s) a(t)} f_{1}(s, t)
$$

for every $s, t \in G^{+}$.

Let $M^{\prime}\left(G^{+}\right)$be the set of all multipliers on $G^{+}$. Then $M^{\prime}\left(G^{+}\right)$is an abelian group under pointwise multiplication. We say that a multiplier $f$ on $G^{+}$is trivial or exact if it is of the form

$$
f(s, t)=a(s+t) / a(s) a(t)
$$

for some measurable $a: G^{+} \rightarrow \mathbf{T}$. The set of all trivial multipliers form a subgroup $M_{0}^{\prime}\left(G^{+}\right)$of $M^{\prime}\left(G^{+}\right)$. Finally, the multiplier group is defined by

$$
M\left(G^{+}\right)=M^{\prime}\left(G^{+}\right) / M_{0}^{\prime}\left(G^{+}\right) .
$$

The group $M(G)$ is defined similarly [7].

We will show that every multiplier on the semigroup $G^{+}$can be extended to a multiplier on the whole group $G$, thus reducing the study of semigroup

Received by the editors April 10, 1991 and, in revised form, July 1, 1991.

1991 Mathematics Subject Classification. Primary 46L55, 47D03; Secondary 20C25. 
multipliers to the study of group multipliers. Our main tool is an algebraic result that states that certain semigroups can be embedded in groups. We begin by summarizing some known results on semigroup theory. The reader can find more detail in [3, Chapter 1]. In accordance with [3], we use algebraists' notations: functions act on the right and compositions of functions go from left to right.

Let $S$ be a multiplicative semigroup that satisfies the cancellation law, has an identity $e$, and has no idempotent other than $e$. By $\mathscr{T}(S)$, we mean the semigroup of all mappings from $S$ into $S$, with composition of functions being the semigroup operation. The regular representation of $S$ is the map $\rho: S \rightarrow \mathscr{T}(S)$ defined by $x \rho_{a}=x a$ for $a, x \in S$. The map $\rho$ is a faithful representation (i.e., a 1-1 homomorphism), so we can identify $S$ with its image $\rho(S)$. Note that each $\rho_{a}$ is $1-1$ since $S$ satisfies the cancellation law.

By $\mathscr{P} \mathscr{T}(S)$, we mean the set of all 1-1 functions from a subset of $S$ onto another subset of $S$. For $\alpha \in \mathscr{P} \mathscr{T}(S)$, we let $\operatorname{dom}(\alpha)$ and $\operatorname{ran}(\alpha)$ be the domain and range of $\alpha$. respectively. The inverse of $\alpha$, denoted $\alpha^{-1}$, is then a 1-1 map from $\operatorname{ran}(\alpha)$ onto $\operatorname{dom}(\alpha)$. We include in $\mathscr{P} \mathscr{T}(S)$ the function that sends the empty set of $S$ onto the empty set of $S$ and denote this function by $\diamond$. Suppose $\alpha, \beta \in \mathscr{P} \mathscr{T}(S)$. If $\operatorname{ran}(\alpha) \cap \operatorname{dom}(\beta) \neq \varnothing$ then we define $\alpha \beta$ to be the usual composition of functions with $\operatorname{dom}(\alpha \beta)=(\operatorname{ran}(\alpha) \cap \operatorname{dom}(\beta)) \alpha^{-1}$. If $\operatorname{ran}(\alpha) \cap \operatorname{dom}(\beta)=\varnothing$, define $\alpha \beta=\diamond$. This operation turns $\mathscr{P} \mathscr{T}(S)$ into a semigroup. In fact, it is an inverse semigroup in the sense that every element $\alpha$ of $\mathscr{P} \mathscr{T}(S)$ has a unique inverse $\beta$, i.e., an element $\beta$ satisfying $\alpha \beta \alpha=\alpha$ and $\beta \alpha \beta=\beta$ (take $\beta=\alpha^{-1}$ ). Note that $\rho(S) \subseteq \mathscr{P T}(S)$.

The conditions we put on $S$ so far are not strong enough to guarantee that $S$ be embedded in a group. What is needed is the following condition

$$
S a \cap S b \neq \varnothing
$$

for every $a, b \in S$. When this condition is satisfied, we embed $S$ in a group as follows. Let $\Sigma$ be the subsemigroup of $\mathscr{P} \mathscr{T}(S)$ generated by $\rho(S)$ and $\rho(S)^{-1}$. Equivalently, $\Sigma$ is the inverse subsemigroup of $\mathscr{P} \mathscr{T}(S)$ generated by $\rho(S)$. When $\alpha, \beta \in \Sigma$, we define $\alpha \sim \beta$ if $\alpha$ and $\beta$ are extensions of some $\gamma \in \Sigma$, i.e., $\operatorname{dom}(\alpha), \operatorname{dom}(\beta) \supseteq \operatorname{dom}(\gamma)$ and $\alpha, \beta$ agree with $\gamma$ on $\operatorname{dom}(\gamma)$. The quotient semigroup $\Sigma / \sim$ with operation $[\alpha][\beta]=[\alpha \beta]$ turns out to be a group. The identity of $\Sigma / \sim$ is $\left[\rho_{e}\right]$. The inverse of $[\alpha]$ is $\left[\alpha^{-1}\right]$.

Condition (4) implies that every element of $\Sigma$ has nonempty domain and range. This fact is used to show that $\rho_{a} \sim \rho_{b}$ iff $a=b$ and hence $S$ is embedded in $\Sigma / \sim$ [3, Theorem 1.23]. Actually there is a smaller subgroup of $\Sigma / \sim$ containing $S$. Let $H$ be the set of all elements of $\Sigma / \sim$ of the form $\left[\rho_{a}\right]^{-1}\left[\rho_{b}\right]$ where $a, b \in S$. Then $H$ is a group containing $S$ [3, Theorem $1.24]$.

A multiplier $f$ on $G^{+}$(resp. $\left.G\right)$ is symmetric if $f(s, t)=f(t, s)$ for every $s, t \in G^{+}$(resp. $\left.G\right)$.

Theorem 1. Every multiplier $f$ on $G^{+}$can be extended to a multiplier on $G$. Furthermore, if $f$ is symmetric then it has a symmetric extension.

Proof. On $S \stackrel{\text { def }}{=} G^{+} \times \mathbf{T}$, define an operation by

$$
(s, \beta)(t, \gamma)=(s+t, \beta \gamma f(s, t)) .
$$


The multiplier condition (1) implies that this operation is associative, hence $S$ is a semigroup. The identity of $S$ is $(0,1)$. It is easy to show that $S$ satisfies the cancellation law and contains no idempotents other than $(0,1)$. Furthermore, condition (4) is satisfied, i.e.,

$$
S(s, \beta) \cap S(t, \gamma) \neq \varnothing
$$

for every $(s, \beta),(t, \gamma) \in S$. Indeed suppose, without loss of generality, that $s \leq t$. Then the above intersection contains the element

$$
(t-s, \gamma \overline{\beta f(t-s, s)})(s, \beta)=(0,1)(t, \gamma) .
$$

It follows that $S$ can be embedded in a group as in the preceding discussion.

We claim that $H=\left\{\left[\rho_{(t, \gamma)}\right],\left[\rho_{(t, \gamma)}^{-1}\right]: t \in G^{+}, \gamma \in \mathbf{T}\right\}$. A typical element of $H$ is of the form $\left[\rho_{(s, \beta)}\right]^{-1}\left[\rho_{(t, \gamma)}\right]=\left[\rho_{(s, \beta)}^{-1} \rho_{(t, \gamma)}\right]$ where $(s, \beta),(t, \gamma) \in S$. The function $\rho_{(s, \beta)}$ has domain $G^{+} \times \mathbf{T}$ and range $(G \cap[s, \infty)) \times \mathbf{T}$ and is given by.

$$
(r, \alpha) \rho_{(s, \beta)}=(r+s, \alpha \beta f(r, s)) .
$$

Its inverse has domain $(G \cap[s, \infty)) \times \mathbf{T}$ and range $G^{+} \times \mathbf{T}$ and is given by

$$
(r, \alpha) \rho_{(s, \beta)}^{-1}=(r-s, \alpha \overline{\beta f(r-s, s)}) \text {. }
$$

Thus $\rho_{(s, \beta)}^{-1} \rho_{(t, \gamma)}$ has domain $(G \cap[s, \infty)) \times \mathbf{T}$ and is given by

$$
\begin{aligned}
(r, \alpha) \rho_{(s, \beta)}^{-1} \rho_{(t, \gamma)} & =(r-s, \alpha \overline{\beta f(r-s, s)}) \rho_{(t, \gamma)} \\
& =(r-s+t, \alpha \bar{\beta} \gamma \overline{f(r-s, s)} f(r-s, t)) .
\end{aligned}
$$

If $t \geq s$ then the multiplier condition (1) implies

$$
\overline{f(r-s, s)} f(r-s, t)=f(r, t-s) \overline{f(s, t-s)},
$$

and so (5) becomes

$$
\begin{aligned}
(r, \alpha) \rho_{(s, \beta)}^{-1} \rho_{(t, \gamma)} & =(r-s+t, \alpha \bar{\beta} \gamma f(r, t-s) \overline{f(s, t-s)}) \\
& =(r, \alpha) \rho_{(t-s, \bar{\beta} \gamma \overline{f(s, t-s)})} .
\end{aligned}
$$

Thus

$$
\rho_{(s, \beta)}^{-1} \rho_{(t, \gamma)} \sim \rho_{(t-s, \bar{\beta} \gamma \overline{f(s, t-s)})}
$$

when $t \geq s$.

If $t<s$ then (1) implies

$$
\overline{f(r-s, s)} f(r-s, t)=f(t, s-t) \overline{f(r-s+t, s-t)}
$$

and (5) becomes

$$
\begin{aligned}
(r, \alpha) \rho_{(s, \beta)}^{-1} \rho_{(t, \gamma)} & =(r-s+t, \alpha \bar{\beta} \gamma f(t, s-t) \overline{f(r-s+t, s-t)}) \\
& =(r, \alpha) \rho_{(s-t, \beta \bar{\gamma} \overline{f(t, s-t)}}^{-1} .
\end{aligned}
$$

Thus

$$
\rho_{(s, \beta)}^{-1} \rho_{(t, \gamma)} \sim \rho_{(s-t, \beta \bar{\gamma}}^{-1} \overline{f(t, s-t))}
$$

when $t<s$. 
We have shown $H \subseteq\left\{\left[\rho_{(t, \gamma)}\right],\left[\rho_{(t, \gamma)}^{-1}\right]: t \in G^{+}, \gamma \in \mathbf{T}\right\}$. The other inclusion follows from $\left[\rho_{(t, \gamma)}\right]=\left[\rho_{(0,1)}\right]^{-1}\left[\rho_{(t, \gamma)}\right]$ and $\left[\rho_{(t, \gamma)}^{-1}\right]=\left[\rho_{(t, \gamma)}\right]^{-1}\left[\rho_{(0,1)}\right]$.

Note that

$$
\begin{aligned}
& {\left[\rho_{(s, \beta)}\right]=\left[\rho_{(t, \gamma)}\right] \quad \text { iff } \quad(s, \beta)=(t, \gamma),} \\
& {\left[\rho_{(s, \beta)}^{-1}\right]=\left[\rho_{(t, \gamma)}^{-1}\right] \quad \text { iff } \quad(s, \dot{\beta})=(t, \gamma) \text {, }} \\
& {\left[\rho_{(s, \beta)}\right]=\left[\rho_{(t, \gamma)}^{-1}\right] \quad \text { iff } \quad s=t=0 \text { and } \beta \gamma=1 \text {. }}
\end{aligned}
$$

Thus, as a set, $H$ can be identified with the set $G \times \mathbf{T}$ (by identifying $\left[\rho_{(t, \gamma)}\right]$ with $(t, \gamma)$ and $\left[\rho_{(t, \gamma)}^{-1}\right]$ with $\left.(-t, \bar{\gamma})\right)$. Since $H$ is a group, the above identification induces a group structure on $G \times \mathbf{T}$. Our goal now is to write out explicitly the multiplication table for $G \times \mathbf{T}$.

The domain of $\rho_{(s, \beta)} \rho_{(t, \gamma)}^{-1}$ is the set of all $(r, \alpha) \in G^{+} \times \mathbf{T}$ such that $r+s \geq t$ and we have

$$
\begin{aligned}
(r, \alpha) \rho_{(s, \beta)} \rho_{(t, \gamma)}^{-1} & =(r+s, \alpha \beta f(r, s)) \rho_{(t, \gamma)}^{-1} \\
& =(r+s-t, \alpha \beta \bar{\gamma} f(r, s) \overline{f(r+s-t, t)}) .
\end{aligned}
$$

It $s \geq t$ then (1) implies

$$
f(r, s) \overline{f(r+s-t, t)}=f(r, s-t) \overline{f(s-t, t)}
$$

and (8) becomes

$$
\begin{aligned}
(r, \alpha) \rho_{(s, \beta)} \rho_{(t, \gamma)}^{-1} & =(r+s-t, \alpha \beta \bar{\gamma} f(r, s-t) \overline{f(s-t, t)} \\
& =(r, \alpha) \rho_{(s-t, \beta \bar{\gamma} \overline{f(s-t, t)})}
\end{aligned}
$$

Thus

$$
\left.\rho_{(s, \beta)} \rho_{(t, \gamma)}^{-1} \sim \rho_{(s-t, \beta \bar{\gamma}} \overline{f(s-t, t)}\right)
$$

when $s \geq t$.

If $s<t$ then (1) implies

$$
f(r, s) \overline{f(r+s-t, t)}=\overline{f(r+s-t, t-s)} f(t-s, s)
$$

and $(8)$ becomes

$$
\begin{aligned}
(r, \alpha) \rho_{(s, \beta)} \rho_{(t, \gamma)}^{-1} & =(r+s-t, \alpha \beta \bar{\gamma} \overline{f(r+s-t, t-s)} f(t-s, s) \\
& =(r, \alpha) \rho_{(t-s, \bar{\beta} \gamma \overline{f(t-s, s)})}^{-1} .
\end{aligned}
$$

Thus

$$
\rho_{(s, \beta)} \rho_{(t, \gamma)}^{-1}=\rho_{(t-s, \bar{\beta} \gamma \overline{f(t-s, s)}}^{-1}
$$

when $s<t$.

Finally we have

$$
\rho_{(s, \beta)} \rho_{(t, \gamma)}=\rho_{(s+t, \beta \gamma f(s, t))}
$$

and

$$
\rho_{(s, \beta)}^{-1} \rho_{(t, \gamma)}^{-1}=\left(\rho_{(t, \gamma)} \rho_{(s, \beta)}\right)^{-1}=\rho_{(t+s, \gamma \beta f(t, s))}^{-1} .
$$


Putting together (6), (7), (9)-(12), we have the following multiplication table for $G \times \mathbf{T}$ :

$$
(s, \beta)(t, \gamma)= \begin{cases}(s+t, \beta \gamma f(s, t)) & \text { if } s \geq 0, t \geq 0, \\ (s+t, \beta \gamma \overline{f(-t,-s)}) & \text { if } s<0, t<0, \\ (s+t, \beta \gamma \overline{f(-s, t+s)}) & \text { if } s<0, t \geq 0, t \geq-s, \\ (s+t, \beta \gamma f(t,-s-t)) & \text { if } s<0, t \geq 0, t<-s, \\ (s+t, \beta \gamma \overline{f(s+t,-t)}) & \text { if } s \geq 0, t<0, s \geq-t, \\ (s+t, \beta \gamma f(-t-s, s)) & \text { if } s \geq 0, t<0, s<-t .\end{cases}
$$

Now define a map $\tilde{f}: G \times G \rightarrow \mathbf{T}$ by the relation

$$
(s, \boldsymbol{\beta})(t, \gamma)=(s+t, \beta \gamma \tilde{f}(s, t))
$$

for $s, t \in G$ and $\beta, \gamma \in \mathbf{T}$. It is clear that $\tilde{f}$ is Borel-measurable and extends $f$. Furthermore, the associativity of the group operation of $G \times \mathbf{T}$ implies that $\tilde{f}$ is a multiplier. If $f$ is symmetric then the group $G \times \mathbf{T}$ is abelian. This implies $\tilde{f}$ is symmetric.

Remarks. As a consequence, we obtain a new proof of Arveson's result that says that every multiplier $f$ on $\mathbf{R}^{+}$is trivial [1, $\left.\S 2\right]$. Indeed this result follows from the above fact that $f$ can be extended to a multiplier on $\mathbf{R}$ and the fact that every multiplier on $\mathbf{R}$ is trivial [7].

Corollary 2. A multiplier $f$ on $G^{+}$is trivial iff it is symmetric.

Proof. Clearly, if $f$ is trivial then it is symmetric. Conversely, suppose that $f$ is symmetric. Then by Theorem 1 , it has a symmetric extension $\tilde{f}$. This implies that $\tilde{f}$ is trivial [6, Lemma 7.2], hence $f$ is trivial.

Theorem 3. The map $[f] \mapsto\left[\left.f\right|_{G^{+} \times G^{+}}\right]$is an isomorphism of $M(G)$ onto $M\left(G^{+}\right)$.

Proof. That the above map is onto follows from Theorem 1. To show that it is $1-1$, assume $\left.f\right|_{G^{+} \times G^{+}}$is trivial. Since every multiplier on $G$ is similar to a bicharacter [6, Theorem 7.1], we may assume that $f$ is a bicharacter. It follows that $f$ is symmetric since $\left.f\right|_{G^{+} \times G^{+}}$is symmetric. Hence $f$ is trivial [6, Lemma 7.2].

\section{ACKNOWLEDGMENT}

The results of this paper are taken from the author's Ph.D. Thesis [5] written under the direction of Professor William Arveson. We thank the referee for pointing out that Chernoff [2] has independently obtained similar results for connected subsemigroups of $\mathbf{R}$.

\section{REFERENCES}

1. W. Arveson, An addition formula for the index of semigroups of endomorphisms of $\mathscr{B}(\mathscr{H})$, Pacific J. Math. 137 (1989), 19-36.

2. P. Chernoff, Extensions and triviality of multipliers on sub-semigroups of the reals, Semigroup Forum 41 (1990), 237-244.

3. A. H. Clifford and G. B. Preston, The algebraic theory of semigroups, Vol. I, Math. Surveys Monographs, vol. 7, Amer. Math. Soc., Providence, RI, 1961. 
4. H. Dinh, Discrete product systems and their $C^{*}$-algebras, J. Funct. Anal. 102 (1991), 1-34. 5. __ Doctoral Dissertation, Univ. of California, Berkeley, 1989.

6. A. Kleppner, Multipliers on abelian groups, Math. Ann. 158 (1965), 11-34.

7. V. S. Varadarajan, Geometry of quantum theory, Springer-Verlag, New York, 1985.

Department of Mathematics, Purdue University, West Lafayette, Indiana 47907 Current address: Mathematics and Computer Science Department, Macalester College, 1600 Grand Avenue, Saint Paul, Minnesota 55105

E-mail address: dinh@math.macalstr.edu 